\title{
PARÂMETROS GENÉTICOS E GANHOS COM A SELEÇÃO DE PROGÊNIES DE Euterpe oleracea NA FASE JUVENIL
}

\author{
João Tomé de Farias Neto' ${ }^{1}$, Maria do Socorro Padilha de Oliveira ${ }^{1}$, \\ Marcos Deon Vilela de Resende ${ }^{2}$, Juliana Chagas Rodrigues ${ }^{3}$
}

(recebido: 4 de janeiro de 2011; aceito: 30 de março de 2012)

\begin{abstract}
RESUMO: São apresentados e discutidos os parâmetros e os ganhos genéticos obtidos, aos 36 meses pós plantio, de progênies de polinização aberta de Euterpe oleracea quanto aos caracteres altura da planta (AP), diâmetro da planta à altura do colo (DPC), número de folhas vivas (NFV) e número de perfilhos (NP), pela metodologia de modelos lineares mistos (procedimento REML/BLUP). As trinta progênies foram avaliadas em blocos casualisados com três repetições e parcelas lineares de cinco plantas, no espaçamento de $6 \mathrm{~m}$ x $4 \mathrm{~m}$. Os valores obtidos de herdabilidade individual $(0,55 ; 0,44 ; 0,38$ e 0,43$)$ e para médias de progênies $(0,64 ; 0,54 ; 0,58$ e 0,64 ) para AP, DPC, NFV e NP, respectivamente, foram expressivos, fato que indica possibilidade de progresso genético com a seleção. A acurácia entre os valores genéticos preditos e os verdadeiros foram de 0,802 para AP, $0,736 \mathrm{DPC}, 0,760$ para NFV e 0,797 para NP. Com exceção do caráter NFV, os valores dos coeficientes de variação genética individual foram altos ( $>10 \%)$, confirmando o potencial da população para a seleção. Ganhos genéticos preditos de $89,3 \%$ foram obtidos para o caráter AP e de $2,1 \%$ para DCP, com a seleção dos vinte indivíduos superiores. Foi detectada correlação entre os caracteres altura e diâmetro da planta. Entre idades, para os mesmos caracteres, foram detectadas correlações fenotípicas positivas de médias magnitudes.
\end{abstract}

Palavras-chave: Herdabilidade, variabilidade, acurácia.

\section{GENETIC PARAMETERS AND SELECTION GAINS FOR Euterpe oleracea IN JUVENILE PHASE}

\begin{abstract}
Genetics parameters and selection gains, obtained 36 months after planting, are presented and discussed for progenies of open pollinated population of açai palm for plant height $(A P)$, plant diameter $(D P C)$, number of live leaves ( NFV) and tiller number (NP), based on the linear mixed model methodology (REML / BLUP). The thirty progenies were evaluated in a randomized blocks design with three replications and plots offive plants, spaced at $6 \mathrm{~m} \times 4 \mathrm{~m}$. The values obtained for individual heritability (0.55, $0.44,0.38$ and 0.43$)$ and for progeny means $(0.64,0.54,0.58$ and 0.64$)$ for $A P, D P C, N F V$ and $N P$, respectively, were expressives, which indicates the possibility of genetic progress with the selection. The accuracy among the genetics values predicted and the true were of 0.802 for height, 0.736 for diameter, 0.760 for number of live leaves and 0.797 for tiller number. With the exception of NFV character, the coefficients of individual genetic variation were high $(>10 \%)$, confirming the potential of the population for selection. Predicted genetic gains of $89.3 \%$ were obtained for the character AP and $2.1 \%$ for DCP, with the selection of the twenty top individuals. Correlation was found between height and diameter of the plant. Among ages, for the same characters, positive correlations of mean magnitudes were found.
\end{abstract}

Key words: Heritability, variability, accuracy.

\section{INTRODUÇÃO}

Euterpe oleracea Mart.(açaizeiro) é uma espécie frutífera e produtora de palmito, nativa da Amazônia sendo o Estado do Pará o maior produtor e o principal consumidor, onde estão estabelecidas densas e diversificadas populações naturais em áreas de várzeas. A maior parte da produção é extrativista, demonstrando que a atividade ainda está muito dependente de E. oleracea nativa.
Com a expansão do mercado nacional e internacional da polpa dessa palmeira além do manejo de áreas de várzeas, está expandindo o plantio em áreas de terra firme, em antigas áreas de Piper nigrum (pimenta- doreino e de lavouras abandonadas. Estes cultivos envolvem consórcios com outras espécies perenes como Theobroma cacao (cacau), Theobroma grandiflorum (cupuaçu), $P$. nigrum etc., como etapa final de cultivos, tais como Passiflora edulis (maracujá), Musa spp. (bananeira) e etc.,

\footnotetext{
${ }^{1}$ Engenheiro Agrônomo, Pesquisador da Embrapa Amazônia Oriental - Travessa Dr. Enéas Pinheiro, s/n. - Cx. P. 48 - 66095-100 - Belém, PA, Brasil-tome@cpatu.embrapa.br, spadilha@cpatu.embrapa.br

${ }^{2}$ Engenheiro Agrônomo, Pesquisador da Embrapa Florestas - Estrada da Ribeira, km 11 - Cx. P. 319 - 83411-000 - Colombo, PR, Brasil marcos.deon@gmail.com

${ }^{3}$ Engenheiro Agrônomo - Universidade Federal Rural da Amazônia/UFRA - Departamento de Agronomia - Cx. P. 917 - 66.077-530 - Belém, PA, Brasil - juliana_c_rodrigues@yahoo.com.br
}

Cerne, Lavras, v. 18, n. 3, p. 515-521, jul./set. 2012 
e também, aproveitando áreas de pastagens degradadas. Nesse sentido, a mesorregião do Nordeste Paraense, tem despertado a atenção dos produtores no plantio de E. oleracea, muitos deles, procurando inovar técnicas visando a desenvolver sistemas de cultivos apropriados, aumentar a produtividade e a produção, tanto na safra como na entressafra (HOMMA et al., 2009). Desse modo, a disponibilização de material genético para um mercado de consumo crescente e adaptado aos sistemas de produção, devem fazer parte dos objetivos do melhoramento genético de E. oleracea.

O programa de melhoramento genético dessa espécie é recente, tendo-se iniciado a partir de 1984 com coletas de sementes em condições de ocorrência natural da espécie (LIMA; COSTA, 1991), com posterior caracterização, avaliação e seleção de plantas superiores. Como resultado desse trabalho foi lançado a primeira cultivar para terra firme, a BRS Pará (OLIVEIRA; FARIAS NETO, 2005). Recentemente, novas coletas foram realizadas e os materiais em avaliação em campo sob a forma de teste de progênies, que se constitui numa das estratégias mais exploradas pelos melhoristas, com o objetivo de estimação de parâmetros genéticos e seleção de indivíduos, procurando avaliar a magnitude e a natureza da variância genética disponível, com vistas a quantificar e maximizar os ganhos genéticos, utilizandose procedimentos de seleção adequados.

Segundo Resende (2002), no melhoramento de espécies perenes, o uso de técnicas de avaliação genética, com base em modelos mistos tipo REML/BLUP (máxima verossimilhança restrita/melhor predição linear não viciada, tende a maximizar os ganhos genéticos obtidos, por se tratar de um procedimento estimativo, especialmente para dados desbalanceados, como no presente estudo, predizendo valores genéticos dos indivíduos em testes de progênies. O procedimento REML/BLUP tem sido usado no melhoramento de palmáceas para a seleção de progênies e indivíduos em $E$. oleracea (FARIAS NETO et al., 2007) e Cocus nucifera (coco) (FARIAS NETO et al., 2009), dentre outros.

Considerando que em programas de melhoramento genético de espécies perenes é importante o conhecimento do controle genético de caracteres de interesse nas diversas etapas de avaliação das progênies com o propósito de definir a melhor estratégia de melhoramento e de ganho genético com a seleção, o presente trabalho foi realizado com os objetivos de estimar na fase juvenil parâmetros genéticos e fenotípicos e de realizar a avaliação e seleção genotípica de progênies e indivíduos de E. oleracea.

\section{MATERIAL E MÉTODOS}

O estudo foi realizado na base experimental da Embrapa Amazônia Oriental localizada no município de Tomé-Açu, no Nordeste paraense, aproximadamente entre as latitudes de $01^{\circ} 57^{\prime} 38^{\prime \prime} \mathrm{S}$ e $03^{\circ} 16^{\prime} 37^{\prime}$ ' $\mathrm{S}$ e as longitudes

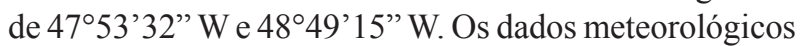
indicam clima quente e úmido, ajustando-se ao tipo climático Ami, da classificação de Köppen (PACHECO; BASTOS, 2001). A precipitação pluviométrica é caracterizada por dois períodos distintos de chuvas, um de dezembro a maio, com índices superiores a $150 \mathrm{~mm} /$ mês, onde se concentram cerca de $80 \%$ do total anual de precipitação, e outro, de junho a novembro, com índice variando, de $49 \mathrm{~mm}$ a $105 \mathrm{~mm}$.

As mudas foram produzidas partindo de sementes coletadas de matrizes nativas de polinização aberta no município de Anajás, PA em maio de 2004. Essa região tem como principal característica a produção de frutos em período considerado como entressafra no estado, ou seja, no primeiro semestre. Na seleção fenotípica das árvores em condições naturais utilizou-se como critérios de seleção o número de cacho por planta, tamanho do cacho, presença de perfilhos e estado fitossanitário das plantas.

$\mathrm{O}$ teste de progênies foi estabelecido em março de 2005, usando o delineamento experimental blocos casualizados, com trinta tratamentos (progênies), três repetições e cinco plantas por parcela no espaçamento de $6 \mathrm{~m} \mathrm{x} 4 \mathrm{~m}$, além de uma bordadura externa ao experimento.

As progênies foram avaliadas em três épocas: março - abril dos anos 2006, 2007 e 2008 em nível de indivíduos para os caracteres: altura da planta - AP (medida em centímetro do solo até o ponto de inserção da folha guia e a primeira folha expandida), diâmetro da planta à altura do colo (DPC), número de folhas vivas (NFV) e número de perfilhos (NP) (somente nos anos de 2006 e 2007).

As estimativas de componentes de variância e parâmetros genéticos foram obtidas pela metodologia de modelos lineares mistos (máxima verossimilhança restrita/ melhor predição linear não viciada), empregando-se o aplicativo computacional genético-estatístico Selegen REML/BLUP, considerando as progênies como sendo de meios-irmãos, delineamento blocos casualizados, com várias plantas por parcela, um só local e uma única população, seguindo o procedimento proposto por Resende (2002):

Cerne, Lavras, v. 18, n. 3, p. 515-521, jul./set. 2012 


$$
\mathrm{y}=\mathrm{Xb}+\mathrm{Za}+\mathrm{Wc}+\mathrm{e},
$$

em que:

y, b, a, c, e - vetores de dados, dos efeitos de bloco (fixos), genéticos aditivos (aleatório), de parcelas (efeitos aleatórios de ambiente comum das parcelas) e de erros aleatórios, respectivamente;

$\mathrm{X}, \mathrm{ZeW}$ - matrizes de incidência para b, a, c, respectivamente.

Associados a esse modelo tem-se as seguintes distribuições e estruturas de médias e variâncias:

$y \mid b, V \sim N(X b, V)$

$a \mid A, \sigma_{a}^{2} \sim N\left(0, A \sigma_{a}^{2}\right)$

$c \mid \sigma_{c}^{2} \sim N\left(0, I \sigma_{c}^{2}\right)$

$e \mid \sigma_{e}^{2} \sim N\left(0, I \sigma_{e}^{2}\right)$

As covariâncias entre todos os efeitos aleatórios do modelo são consideradas nulas, ou seja, $\operatorname{Cov}(a, c)=0$, $\operatorname{Cov}\left(a, e^{\prime}\right)=0$ e $\operatorname{Cov}\left(c, e^{\prime}\right)=0$.

Assim:

$E\left[\begin{array}{c}y \\ a \\ c \\ e\end{array}\right]=\left[\begin{array}{c}X b \\ 0 \\ 0 \\ 0\end{array}\right]$ Var $\left[\begin{array}{c}y \\ a \\ c \\ e\end{array}\right]=\left[\begin{array}{cccc}V & Z G & W C & R \\ G Z^{\prime} & G & 0 & 0 \\ C W^{\prime} & 0 & C & 0 \\ R & 0 & 0 & R\end{array}\right]$,

em que:

$G=A \sigma_{A}^{2}$

$C=I \sigma_{c}^{2}$

$R=I \sigma_{e}^{2}$

$V=Z A \sigma_{a}^{2} Z^{\prime}+W I \quad \sigma_{c}^{2} W^{\prime}+I \quad \sigma_{e}^{2}=Z G Z^{\prime}+W C W^{\prime}+R$.

Equações de modelo misto:

$\left[\begin{array}{l}b \\ \hat{a} \\ \hat{c}\end{array}\right]=\left[\begin{array}{ccc}X^{\prime} X & X^{\prime} Z & X^{\prime} W \\ Z^{\prime} X & Z^{\prime} Z+A^{-1} \lambda_{1} & Z^{\prime} W \\ W^{\prime} X & W^{\prime} Z & W^{\prime} W+I \lambda_{2}\end{array}\right]^{-1}\left[\begin{array}{c}X^{\prime} y \\ Z^{\prime} y \\ W^{\prime} y\end{array}\right]$,

em que:

$\lambda_{1}=\frac{1-h^{2}-c^{2}}{h^{2}}=\frac{\sigma_{e}^{2}}{\sigma_{a}^{2}} ; \quad \lambda_{2}=\frac{1-h^{2}-c^{2}}{c^{2}}=\frac{\sigma_{e}^{2}}{\sigma_{c}^{2}}$

$h^{2}=\frac{\sigma_{a}^{2}}{\sigma_{a}^{2}+\sigma_{c}^{2}+\sigma_{e}^{2}}$ : herdabilidade individual no sentido restrito. $c^{2}=\sigma_{c}^{2} /\left(\sigma_{a}^{2}+\sigma_{c}^{2}+\sigma_{e}^{2}\right):$ correlação devida ao ambiente comum da parcela.

$\sigma_{a}^{2}$ : variância genética aditiva.

$\sigma_{c}^{2}$ : variância entre parcelas.

$\sigma_{e}^{2}$ : variância residual (ambiental dentro de parcelas + não aditiva).

A: matriz de correlação genética aditiva entre os indivíduos em avaliação.

As estimativas dos parâmetros genéticos foram obtidas pelo procedimento REML partindo de iterações nas equações de modelo misto.

\section{RESULTADOS E DISCUSSÃO}

As herdabilidades individuais no sentido restrito para os caracteres podem ser considerados de média a alta magnitudes $(0,56 ; 0,44 ; 0,38$ e 0,43$)$ para AP, DPC, NFV e NP, respectivamente (Tabela 1). Valores mais expressivos foram obtidos para média de progênies $(0,64 ; 0,54 ; 0,58 \mathrm{e}$ $0,64)$. Esses resultados são superiores àqueles estimados por Farias Neto et al. (2007), que avaliaram a mesma espécie, mas com procedência dos municípios de Afuá e Chaves e indicam a possibilidade de ganhos genéticos consideráveis em resposta à seleção, desde que mesclando informações tanto de progênies quanto dos indivíduos.

Os coeficientes de variação genética individual que expressam em percentagem da média geral a quantidade de variação genética existente, tiveram valores expressivos para os caracteres AP e NP $(21,52$ e 38,03, respectivamente) e moderados para DPC e NFV $(11,52$ e 7,63, respectivamente). Esses resultados sugerem que a população estudada pode ser considerada um conjunto geneticamente promissor para o programa de melhoramento genético de E. oleracea, sendo possível a obtenção de ganhos expressivos via seleção. Vários estudos com essa espécie têm evidenciado a presença de considerável variabilidade genética para vários caracteres, sendo promissoras as possibilidades de melhoramento desses materiais e confirmando as informações no presente ensaio (FARIAS NETO et al., 2008; OLIVEIRA; FARIAS NETO, 2008).

Os coeficientes de variação experimental $\left(\mathrm{CV}_{\mathrm{e}}\right)$ com valores de $13,89 \%$ para AP, $9,02 \%$ para DPC e $5,63 \%$ para NFV, podem ser considerados de baixa magnitude e semelhantes aos estimados por Farias Neto et al. (2007), que obtiveram valores que oscilaram entre 25,62 a $35,21 \%$. Apenas o resultado para o caráter NP $(24,98 \%)$ foi semelhante ao obtido por Farias Neto et al. (2007).

Cerne, Lavras, v. 18, n. 3, p. 515-521, jul./set. 2012 
Tabela 1 - Estimativas de parâmetros genéticos para os caracteres altura da planta (AP), diâmetro da planta à altura do colo (DPC), número de folhas vivas (NFV) e número de perfilhos (NP).

Table 1 -Estimates of genetic parameters for the characters: plant height (AP), diameter (DPC), number of live leaves (NFV) and tiller number (NP).

\begin{tabular}{|c|c|c|c|c|}
\hline Parâmetros Genéticos & $\mathrm{AP}(\mathrm{cm})$ & $\mathrm{DPC}(\mathrm{cm})$ & NFV & NP \\
\hline Variância genética aditiva $\left(\sigma_{a}^{2}\right)$ & 3128,06 & 2,48 & 0,63 & 3,4 \\
\hline Variância ambiental entre parcelas $\left(\sigma_{c}^{2}\right)$ & 683,73 & 0,71 & 0,06 & 0,06 \\
\hline Variância ambiental dentro de parcelas $\left(\sigma_{e}^{2}\right)$ & 753,31 & 2,45 & 0,96 & 4,56 \\
\hline Variância fenotípica individual $\left(\sigma_{F_{i}}^{2}\right)$ & 4565,09 & 5,64 & 1,64 & 8,08 \\
\hline Herdabilidade individual no sentido restrito $\left(h_{i}^{2}\right)$ & 0,55 & 0,44 & 0,38 & 0,43 \\
\hline Herdabilidade da média de progênie $\left(h_{\bar{p}}^{2}\right)$ & 0,64 & 0,54 & 0,58 & 0,64 \\
\hline Acurácia na seleção de progênie $\left(\sqrt{ }\left(h_{\bar{p}}^{2}\right)\right)$ & 0,802 & 0,736 & 0,760 & 0,797 \\
\hline Coeficiente de variação genética individual $\left(C V_{g_{i}} \%\right)$ & 21,52 & 11,35 & 7,63 & 38,03 \\
\hline Coeficiente de variação genética entre progênies $\left(C V_{g_{p}} \%\right)$ & 10,7 & 5,67 & 3,81 & 19,01 \\
\hline Coeficiente de variação residual $\left(C V_{e}^{\%}\right)$ & 13,89 & 9,03 & 5,64 & 24,99 \\
\hline Média $(\bar{Y})$ & 259,91 & 13,87 & 10,36 & 4,89 \\
\hline
\end{tabular}

Nas Tabelas 2 e 3, encontram-se os valores fenotípicos, genéticos aditivos dos vinte melhores indivíduos, ganhos genéticos preditos e a nova média da população para os caracteres AP e DAP, respectivamente. A seleção para AP das 20 progênies selecionadas proporcionou um ganho genético de $89,29 \%$, elevando a média do caráter de $259 \mathrm{~cm}$ para $349 \mathrm{~cm}$ e envolveu 10 progênies de um total de 30 avaliadas. Para o mesmo número de progênies selecionadas para DPC, o ganho foi de $2,12 \mathrm{~cm}$, elevando-se a média da população de 13,87 para 15,99 , contemplando 12 progênies. Comparando-se os resultados obtidos para AP e DPC houve uma predominância da progênie de número 17. Entretanto, há necessidade de avaliações ao longo do programa de melhoramento como forma de confirmar ou não o potencial desses indivíduos para efeito de seleção. Os altos valores de acurácia seletiva para $\mathrm{AP}(0,802)$ e DPC $(0,736)$ reforçam a importância do uso da informação de progênies.

As estimativas de correlação genética entre os caracteres evidencia a alta magnitude encontrada entre AP e DPC $(0,83)$ e as baixas entre AP e DPC com NFV (Tabela 4). A correlação alta e positiva entre AP e DPC indica que a seleção em qualquer um deles poderá proporcionar alterações favoráveis no outro e, consequentemente, na produtividade de frutos, uma vez que esses três caracteres apresentam correlação positiva em palmaceas (OLIVEIRA et al., 2000). Entretanto, estudos sobre seleção que procuram determinar a confiabilidade de um caráter avaliado na fase juvenil como indicador do desempenho produtivo do indivíduo na fase adulta deve ser melhor investigado em E. oleracea.

Os caracteres AP, DPC e NFV mostraram-se negativamente associados com o NP, fato que segundo Bovi et al. (1992), evidencia uma relação de competição dentro da touceira, ou seja, plantas mais perfilhadas possuem menor desenvolvimento da planta mãe, uma vez que estes concorrem com a distribuição de assimilados dentro da touceira. Portanto, essas estimativas permitem fundamentar a ideia da facilidade relativa de se selecionar plantas produtivas, altas e com maior diâmetro; todavia,

Cerne, Lavras, v. 18, n. 3, p. 515-521, jul./set. 2012 
Tabela 2 - Valores fenotípicos, genéticos aditivos e ganhos genéticos preditos dos vinte melhores indivíduos/progênies para o caráter altura da planta $(\mathrm{cm})$.

Table 2 - Phenotypic values, genetic additives and genetic gains predicted of twenty best individuals/progenies for the character plant height $(\mathrm{cm})$.

\begin{tabular}{|c|c|c|c|c|c|}
\hline Progênie & Indivíduo & Valores fenotípicos & $\begin{array}{l}\text { Valores genéticos } \\
\qquad(\mathrm{m}+\mathrm{a})^{*}\end{array}$ & Ganho genético & $\begin{array}{c}\text { Nova média da } \\
\text { população }\end{array}$ \\
\hline 17 & 5 & 435 & 377,43 & 117,52 & 377,43 \\
\hline 2 & 4 & 420 & 374,69 & 116,15 & 376,06 \\
\hline 4 & 5 & 450 & 367,20 & 113,19 & 373,11 \\
\hline 11 & 3 & 365 & 363,55 & 110,81 & 370,72 \\
\hline 2 & 2 & 350 & 362 & 109,20 & 369,14 \\
\hline 17 & 1 & 415 & 360 & 107,8 & 367,73 \\
\hline 1 & 4 & 395 & 356 & 106,3 & 366,18 \\
\hline 5 & 3 & 380 & 354 & 104,8 & 364,69 \\
\hline 21 & 5 & 405 & 349 & 103,1 & 362,99 \\
\hline 11 & 1 & 390 & 347 & 101,5 & 361,44 \\
\hline 17 & 3 & 325 & 343 & 99,9 & 359,83 \\
\hline 21 & 5 & 385 & 341 & 98,4 & 358,29 \\
\hline 1 & 3 & 400 & 339,67 & 96,9 & 356,86 \\
\hline 9 & 3 & 400 & 339,60 & 95,7 & 355,63 \\
\hline 2 & 5 & 400 & 339,34 & 94,6 & 354,54 \\
\hline 3 & 2 & 385 & 336,19 & 93,48 & 353,39 \\
\hline 17 & 2 & 380 & 335,81 & 92,44 & 352,36 \\
\hline 11 & 4 & 370 & 331,94 & 91,31 & 351,22 \\
\hline 1 & 5 & 310 & 331,36 & 90,27 & 350,18 \\
\hline 19 & 3 & 390 & 330,69 & 89,29 & 349,21 \\
\hline
\end{tabular}

$*(\mu+a)=$ valor genético aditivo predito

Tabela 3 - Valores fenotípicos, genéticos aditivos e ganhos genéticos preditos dos vinte melhores indivíduos/progênies para o caráter diâmetro da planta a altura do colo $(\mathrm{cm})$.

Table 3 - Phenotypic values, genetic additives and genetic gains predicted of twenty best individuals/progenies for the character diameter of the plant $(\mathrm{cm})$.

\begin{tabular}{cccccc}
\hline Progênie & Indivíduo & Valores fenotípicos & $\begin{array}{c}\text { Valores genéticos } \\
(\mathrm{m}+\mathrm{a})^{*}\end{array}$ & $\begin{array}{c}\text { Ganho genético } \\
(\mathrm{cm})\end{array}$ & $\begin{array}{c}\text { Nova média da } \\
\text { população }\end{array}$ \\
\hline 17 & 5 & 20,06 & 16,71 & 2,83 & 16,71 \\
6 & 1 & 20,38 & 16,46 & 2,72 & 16,58 \\
17 & 1 & 19,42 & 16,36 & 2,64 & 16,51 \\
11 & 3 & 17,83 & 16,23 & 2,57 & 16,44 \\
1 & 3 & 17,83 & 16,22 & 2,53 & 16,40 \\
9 & 5 & 20,06 & 16,21 & 2,50 & 16,37 \\
\hline & & & & Continua... \\
& & & & To be continued...
\end{tabular}

Cerne, Lavras, v. 18, n. 3, p. 515-521, jul./set. 2012 
Tabela 3 - Continuação...

Table 3 - Continued..

\begin{tabular}{cccccc}
\hline Progênie & Indivíduo & Valores fenotípicos & $\begin{array}{c}\text { Valores genéticos } \\
(\mathrm{m}+\mathrm{a})^{*}\end{array}$ & $\begin{array}{c}\text { Ganho genético } \\
(\mathrm{cm})\end{array}$ & $\begin{array}{c}\text { Nova média da } \\
\text { população }\end{array}$ \\
\hline 23 & 1 & 19,42 & 16,19 & 2,47 & 16,34 \\
23 & 3 & 20,71 & 16,18 & 2,45 & 16,32 \\
1 & 5 & 17,52 & 1609 & 2,43 & 16,29 \\
17 & 2 & 18,47 & 16,02 & 2,40 & 16,27 \\
24 & 2 & 19,42 & 15,91 & 2,37 & 16,23 \\
30 & 4 & 18,47 & 15,86 & 2,34 & 16,20 \\
18 & 3 & 18,78 & 15,76 & 2,30 & 16,17 \\
21 & 5 & 18,78 & 15,73 & 2,27 & 16,14 \\
10 & 1 & 18,15 & 15,72 & 2,24 & 16,11 \\
17 & 4 & 17,83 & 15,67 & 2,21 & 16,08 \\
2 & 4 & 18,47 & 15,66 & 2,19 & 16,06 \\
18 & 3 & 17,83 & 15,59 & 2,16 & 16,03 \\
17 & 2 & 18,47 & 15,59 & 2,14 & 16001 \\
\hline
\end{tabular}

$*(\mu+a)=$ valor genético aditivo predito

Tabela 4 - Coeficientes de correlação genética entre altura da planta (AP), diâmetro da planta (DP), número de folhas vivas (NFV) e número de perfilhos (NP).

Table 4 - Genetic correlation coefficients between plant height $(A P)$, diameter of the plant $(D P)$, number of alive leaves $(N F V)$ and number of tillers (NP).

\begin{tabular}{lcccc}
\hline Caráter & AP & DPC & NFV & NP \\
\hline AP & 1 & 0,83 & 0,14 & $-0,268$ \\
DPC & & 1 & 0,3011 & $-0,1661$ \\
NFV & & & 1 & $-0,2284$ \\
\hline
\end{tabular}

é relativamente difícil adicionar emissão precoce de perfilhos a esse conjunto de caracteres favoráveis em uma mesma planta. Por outro lado, a emissão de perfilhos tem sido apontado como importante caráter no aumento da produtividade de frutos, permitindo inclusive a exploração contínua das touceiras (OHASHI; KAGEYAMA, 2004). Estudos que comprovem a importância da correlação positiva da emissão precoce de perfilhos frente à correlação positiva entre os caracteres altura e diâmetro das plantas na fase juvenil com a produtividade na fase adulta, ainda não existem em E. oleracea.

A avaliação das propriedades genéticas das características de crescimento nos primeiros anos de desenvolvimento é de grande valia para o melhorista que objetiva, além da identificação dos melhores indivíduos, a quantificação da eficiência da seleção precoce (KAGEYAMA, 1983).

As estimativas das correlações genéticas e fenotípicas entre os caracteres AP e DPC nos três primeiros anos de avaliação (Tabela 5) foram positivas para os dois caracteres. Tais correlações indicam que plantas com maior AP e DPC, em idades precoces, tendem a repetir o desempenho em idades mais avançadas, sugerindo que os caracteres apresentam repetibilidade e que existem possibilidades de sucesso com a seleção em idades precoces. Resultados contrários foram obtidos por Ohashi e Kageyama (2004), que encontraram correlações fenotípicas negativas entre as diversas idades de desenvolvimento de E. oleracea.

Os coeficientes de correlação genética foram mais elevados quando envolveram a primeira (um ano pós plantio) e a segunda avaliação (dois anos pós-plantio) quando comparados com as estimativas entre a primeira e terceira avaliação, ou seja, correlações foram superiores entre idades próximas relativamente as idades distantes. È importante salientar que as variações encontradas entre as diferentes idades podem ser atribuídas ao efeito de ano (condição climática diferenciada em cada ciclo de crescimento), efeito de idade propriamente dita (efeito genético) e à interação entre esses efeitos.

Cerne, Lavras, v. 18, n. 3, p. 515-521, jul./set. 2012 
Tabela 5 - Coeficientes de correlação genética e fenotípica entre altura da planta (AP) e diâmetro da planta a altura do colo (DPC) entre os três anos de avaliação.

Table 5-Genetic and phenotypic correlation coefficients between plant height $(A P)$ and diameter of plant height (DPC) among the three years of evaluation.

\begin{tabular}{cccccc}
\hline $\begin{array}{c}\text { Caráter/ } \\
\text { Idadade }\end{array}$ & AP2 & AP3 & $\begin{array}{c}\text { Caráter/ } \\
\text { Idade }\end{array}$ & DPC2 & DPC3 \\
\hline \multirow{2}{*}{ AP1 } & $0,8338^{*}$ & 0,5982 & DPC1 & 0,8086 & 0,2525 \\
& $0,4842^{* *}$ & 0,7235 & & 0,5478 & 0,6461 \\
\hline \multirow{2}{*}{ AP2 } & & 0,7524 & DPC2 & & 0,5396 \\
& & 0,6709 & & & 0,6553 \\
\hline
\end{tabular}

Para cada par de caracteres, o primeiro valor se refere ao coeficiente de correlação genética e o segundo ao coeficiente de correlação fenotípica.

\section{CONCLUSÕES}

A população apresenta alta variabilidade genética evidenciada pelas estimativas de herdabilidade individual associados à média de progênies e dos coeficientes de variação genética.

A continuidade das avaliações em idades mais avançadas, explorando a variabilidade tanto ao nível de indivíduos quanto de progênies permitirá a obtenção de altos ganhos genéticos para os caracteres avaliados.

As correlações estimadas entre os caracteres nas mesmas e nas diferentes idades mostram a possibilidade de se obterem ganhos genéticos pela seleção indireta e precoce.

\section{REFERÊNCIAS}

BOVI, M. L. A.; SAES, L. A.; GODOY JUNIOR, G. Correlações fenotípicas entre caracteres não destrutíveis e palmito em pupunheira. Turrialba, San José, v. 42, n. 3, p. 382-390, 1992.

FARIAS NETO, J. T. de; LINS, P. M. P.; RESENDE, M. D. V. de; MULLER, A. A. Seleção genética em progênies híbridas de coqueiro. Revista Brasileira de Fruticultura, Jaboticabal, v. 31, p. 190-196, 2009.

FARIAS NETO, J. T. de; RESENDE, M. D. V. de; OLIVEIRA, M. S. P. de; NOGUEIRA, O. L.; FALCÃO, P. N. B.; SANTOS, N. S. A. Estimativas de parâmetros genéticos e ganhos de seleção em progênies de polinização aberta de açaizeiro. Revista Brasileira de Fruticultura, Jaboticabal, v. 30, p. 1051-1056, 2008.
FARIAS NETO, J. T. de; RESENDE, M. D. V. de; OLIVEIRA, M. S. P. de; SANTOS, N. S. A.; CANUTO, E. L.; NOGUEIRA, O. L.; MULLER, A. A. Avaliação genética de progênies de polinização aberta de açaí (Euterpe oleracea) e estimativas de parâmetros genéticos. Cerne, Lavras, v. 13, n. 4, p. 376-383, 2007.

HOMMA, A. K. O.; CARVALHO, J. E. U.; MENEZES, A. J. E. A.; FARIAS NETO, J. T.; MATOS, G. B. Custo operacional de açaizeiro irrigado com microaspersão no município de Tomé-Açu. Belém: EMBRAPA Amazônia Oriental, 2009. 5 p. (Comunicado Técnico, 88).

KAGEYAMA, P. Y. Seleção precoce a diferentes idades em progênies de Eucalyptus grandis (Hill) Maiden. 1983. 147 f. Tese (Livre Docência) - Escola Superior de Agricultura "Luiz de Queiroz", Piracicaba, 1983.

LIMA, R. R.; COSTA, J. P. C. Registro de introdução de plantas de culturas pré-colombiana coletadas na Amazônia Brasileira. Belém: EMBRAPA Amazônia Oriental, 1991. 210 p. (Documentos, 58).

OHASHI, S. T.; KAGEYAMA, P. Y. Variabilidade genética entre populações de açaizeiro (Euterpe oleracea Mart.) do estuário amazônico. In: MOURÃO, L.; JARDIM, M. A.; GROSSMANN, M. (Ed.). Açai: possibilidade e limites em processos de desenvolvimento sustentável no estuário amazônico. Belém: CEJUP, 2004. p. 11-26.

OLIVEIRA, M. S. P. de; FARIAS NETO, J. T. Cultivar BRS - Pará: açaizeiro para produção de frutos em terra firme. Belém: EMBRAPA Amazônia Oriental, 2005. 2 p. (Comunicado Técnico, 114).

OLIVEIRA, M. S. P. de; FARIAS NETO, J. T. Seleção massal em açaizeiro para a produção de frutos. Revista de Ciências Agrárias, Teresina, n. 49, p. 145-156, 2008.

OLIVEIRA, M. S. P. de; LEMOS, M. A.; SANTOS, V. F. dos; SANTOS, E. O. dos. Correlação fenotípica entre caracteres vegetativos e de produção de frutos em açaizeiro. Revista Brasileira de Fruticultura, Jaboticabal, v. 22, n. 1, p. 1-5, 2000.

PACHECO, N. A.; BASTOS, T. X. Caracterização climática do município de Tomé-Açu, PA. Belém: EMBRAPA Amazônia Oriental, 2001. 18 p. (Documentos, 87).

RESENDE, M. D. V. Genética biométrica e estatística no melhoramento de plantas perenes. Brasília: EMBRAPA Informação Tecnológica, 2002. 975 p.

Cerne, Lavras, v. 18, n. 3, p. 515-521, jul./set. 2012 
